\title{
Rydberg states of zinc and measurement of the dipole polarizability of the $\mathrm{Zn}^{+}$ion
}

\author{
M. Kompitsas, C. Baharis, and Z. Pan \\ National Hellenic Research Foundation, Theoretical and Physical Chemistry Institute, \\ 48, Vasileos Constantinou Avenue, 11635 Athens, Greece
}

Received April 27, 1993; revised manuscript received November 22, 1993

\begin{abstract}
We have recorded the $4 s n s{ }^{1} S_{0}(n=14-31)$, $4 s n d{ }^{1} D_{2}(n=12-35), 4 s n f^{1,3} F_{J}^{0}(n=8$ to $n=26)$, and $4 s n p^{3} P_{0,1,2}^{0}(n=10$ to $n=40)$ Rydberg series of $\mathrm{Zn}$. We applied various excitation schemes that use two and three laser beams interacting with the $\mathrm{Zn}$ vapor in a heat-pipe-like oven with subsequent thermionic detection of the excited states. From the quantum defects of the nonpenetrating $4 s n f$ levels we estimated the effective dipole polarizability of the $\mathrm{Zn}^{+}$core to be $\alpha=2.30 \pm 0.12 \times 10^{-24} \mathrm{~cm}^{3}$.
\end{abstract}

\section{INTRODUCTION}

This paper deals with systematic measurements of Rydberg states of $\mathrm{Zn}$ below the first ionization limit. The $\mathrm{Zn}$ ground state $4 s^{2}$ consists of two valence electrons outside closed shells. Thus it is homologous to the alkaline-earth atom $\mathrm{Ca}$. In contrast to $\mathrm{Ca}$, the $3 d$ subshell of $\mathrm{Zn}$ is filled with 10 electrons. This results in significant differences in both the bound and the autoionizing spectra of the two atoms. For example, the $\mathrm{Ca}$ doubly excited configurations $3 d 5 s$ and $3 d^{2}{ }^{1} D_{2}$ are embedded among the principal series, and they perturb it. ${ }^{1}$ This fact enables one to study how the interelectronic correlations manifest themselves in the bound spectrum of $\mathrm{Ca}$. Obviously, this is not the case for $\mathrm{Zn}$. Another perturbing configuration ${ }^{2}$ in the bound spectrum of $\mathrm{Ca}$ is $4 p^{2}{ }^{1} S_{0}$. From early research ${ }^{3}$ it is known that the corresponding states for $\mathrm{Zn}$ are autoionizing just above the threshold. From our present measurements it is seen that these states can have only a minor effect on the energies of the bound states of the same symmetry.

The first systematic studies on the bound series of $\mathrm{Zn}$ involved absorption of $\mathrm{Zn}$ atoms in the ground state of light from background continua ${ }^{4}$ (microwave-excited raregas lamps). The principal $4 s n p{ }^{1} P_{1}^{0}$ series was observed up to $n=66$, and from these data the ionization limit was determined to be $75769.33 \mathrm{~cm}^{-1}$. In the same study the observation of the intercombination lines $4 s n p^{3} P_{1}^{0}$ to $n=12$ indicates a slight departure from the pure $L S$ coupling scheme for the $\mathrm{Zn}$ atom. Further weak singlephoton transitions ${ }^{4}$ to $4 s n d{ }^{1} D_{2}$ (for $n \leq 20$ ) have been attributed to quadrupole transitions.

One should expect that the development of the pulsed dye laser, in combination with multistep excitation schemes, would make detailed studies of Rydberg series possible when the angular momentum $J$ is different from 1 , as is the case for the alkaline-earth atoms. The lack of such measurements may be due to the high ionization threshold of $\mathrm{Zn}$ and to the fact that the $4 s 4 p{ }^{1} P_{1}^{0}$ resonance transition lies so high that UV tunable radiation near $214 \mathrm{~nm}$ is necessary for the first step excitation.

With other than conventional absorption spectroscopy, energy levels and lifetimes of the lowest members of the $4 s n s{ }^{1,3} S$ and $4 s n d{ }^{1,3} \mathrm{D}$ series have been deduced. Either these levels were populated by an electron impact, ${ }^{5}$ or the $4 s 4 p^{1} P_{1}^{0}$ level was populated in a HF discharge and then a laser induced the second step transition. ${ }^{6}$ Subsequent decay to the $4 s 4 p{ }^{1} P_{1}^{0}$ level was used for detection. It was claimed ${ }^{6}$ that because of the mixing of the autoionizing $4 p^{2}$ configuration with the series studied, the transition matrix element to the $4 s 4 p$ state decreases rapidly, so that for higher $n$ values no detection was possible.

In the present study we overcome the above problems by using a different excitation and detection method, thus extending the measurements to $n=31$ for the $4 s n s{ }^{1} S_{0}$ and to $n=35$ for the $4 s n d{ }^{1} D_{2}$ states. The $4 s 4 p{ }^{1} P_{1}^{0}$ state was populated through the decay of the higher-lying $4 s 5 s{ }^{1} S_{0}$ state, which was first populated in a two-photon process. To permit detection of the final $4 s n s$ or $4 s n d$ state, the atom is ionized either by an ionizing collision or by absorption of a further photon, and the ion is detected in a thermionic detector. The reduced radiative decay rate $^{6}$ for higher $n$ favors this detection technique. We have also observed the $4 s n f^{1,3} F_{J}^{0}$ states that were previously unknown, and from their energy positions we have estimated the dipole polarizability $\alpha$ of the $\mathrm{Zn}^{+}$core. Finally, we have recorded the $n p^{3} P_{J}^{0}$ levels in a three-laser experiment by means of the $4 p{ }^{3} P_{1}^{0}$ and $5 s{ }^{3} S_{1}$ intermediates.

\section{EXPERIMENTAL TECHNIQUE}

In this experiment, starting from the atomic ground state, the triplet $4 s n p{ }^{3} P_{J}^{0}$, the singlet $4 s n s{ }^{1} S_{0}$, $4 s n d{ }^{1} D_{2}$, and $4 s n f{ }^{1,3} F_{J}^{0}$ Rydberg series have been recorded and their level energies measured.

Two laser systems were employed. They were driven by a common $10-\mathrm{Hz}$ pulse generator, and the time delay and the sequence of their pulses could be set electronically within a few nanoseconds. The first laser system consisted of a Quantel Nd:YAG laser pumping a Quantel TDL50 dye laser, delivering 8-ns pulses in the visible and near-UV region of the spectrum. The second system con- 
sisted of a Lumonics excimer laser pumping a Lumonics EPD 300 dye laser. For the three-laser measurements the excimer output was split to pump a home-built dye laser also; which consisted of a Litmann-type oscillator and an amplifier. The system delivered 8-ns pulses with a bandwidth of $0.5 \mathrm{~cm}^{-1}$.

Different types of excitation method involving two- and three-step excitation schemes have been applied, while the laser inducing the last step has been tuned across the spectrum. For wavelength calibration a fraction of the final laser output was sent into a commercial hollowcathode lamp, and the $\mathrm{Ne}$ optogalvanic spectrum ${ }^{7}$ was simultaneously recorded. For calibration between the $\mathrm{Ne}$ lines another fraction was sent into an evacuated Fabry-Perot interferometer with a $1-\mathrm{cm}^{-1}$ free spectral range, and the transmission fringes were recorded. This method permitted an absolute accuracy of $0.2 \mathrm{~cm}^{-1}$ for the final level energy.

The experimental setup is illustrated in Fig. 1. The atomic vapor was confined in a heat-pipe-like oven, which also served as the detector, operating as a thermionic diode. The $\mathrm{Zn}$ oven consisted of a cylindrical stainlesssteel tube, $42 \mathrm{~cm}$ long with a $33-\mathrm{mm}$ internal diameter. The heated zone in the middle was $15 \mathrm{~cm}$ long, and the temperature was $420-460^{\circ} \mathrm{C}$, controlled by a $\mathrm{NiCr}-\mathrm{Ni}$ thermocouple. Two brass cylinders were mounted at both ends of the tube and sealed off by $O$ rings for vacuum purposes. On these cylindrical blocks the entrance windows for the lasers and tap-water tubing for cooling were mounted. On the same blocks the vacuum pipes and the buffer gas line were connected. A few millibars ( $\left.1 \mathrm{mbar}=7.5 \times 10^{-1} \mathrm{Torr}\right)$ of Ar buffer gas, measured by an MKS Baratron gauge PDR-C-2, was used to reduce vapor diffusion and protect the windows. No systematic shift of the level energies has been detected for this buffer-gas pressure.
The ionization detector consisted of a single stainlesssteel wire (34 $\mathrm{cm}$ long and $0.3 \mathrm{~mm}$ in diameter) stretched off-axis along the tube, operating in the space-chargelimited regime. It was found that at the oven temperature used the wire was not heated enough, and this resulted in a weak signal. So the wire was directly heated by a current of $0.5-1 \mathrm{~A}$. It turned out that the signal was highly sensitive to this current value, that is, to the wire temperature. By a second circuit the wire was negatively biased at $0.8-1.5 \mathrm{~V}$ through a $100-\mathrm{k} \Omega$ resistor while the tube was grounded. A 20-pF capacitor was used to couple the signal to a linear gate (ORTEC Model 9415), and its output was sent to the chart recorder. The signal was further optimized by positioning of the laser beam $\sim 9 \mathrm{~mm}$ from the wire.

\section{MEASUREMENTS AND RESULTS}

A. Singlet 4sns and 4snd Spectra

For excitation of the $4 s n{ }^{1} S_{0}$ and $4 s n d{ }^{1} D_{2}$ states a twostep excitation scheme was applied (see Fig. 2). To populate the $4 s 4 p^{1} P_{1}^{0}$ state (at $46745.4 \mathrm{~cm}^{-1}$ ) in the first step, a wavelength near $213 \mathrm{~nm}$ was needed, which was not available. Therefore we populated this state by first exciting the higher-lying $4 s 5 \mathrm{~s}{ }^{1} S_{0}\left(\right.$ at $55789.2 \mathrm{~cm}^{-1}$ ) in a two-photon nonresonant process $\left(\lambda_{1}=3585 \AA\right.$ ), which decayed radiatively to the $4 s 5 p{ }^{1} P_{1}^{0}$ state. The TDL50 dye-laser beam was set at $7170 \AA$, frequency doubled in a KDP crystal, and sent, slightly focused, into the $\mathrm{Zn}$ oven. The EPD 300 dye-laser beam, counterpropagating to the first beam, was tuned unfocused in the 356-345$\mathrm{nm}$ region to induce the second single-photon transition. A delay of $4 \mathrm{~ns}$ between the two laser pulses was set by optimization of the signal.

In Table 1 the energy values of the observed Rydberg series are listed. For the $n s{ }^{1} S_{0}$ states, new level energy

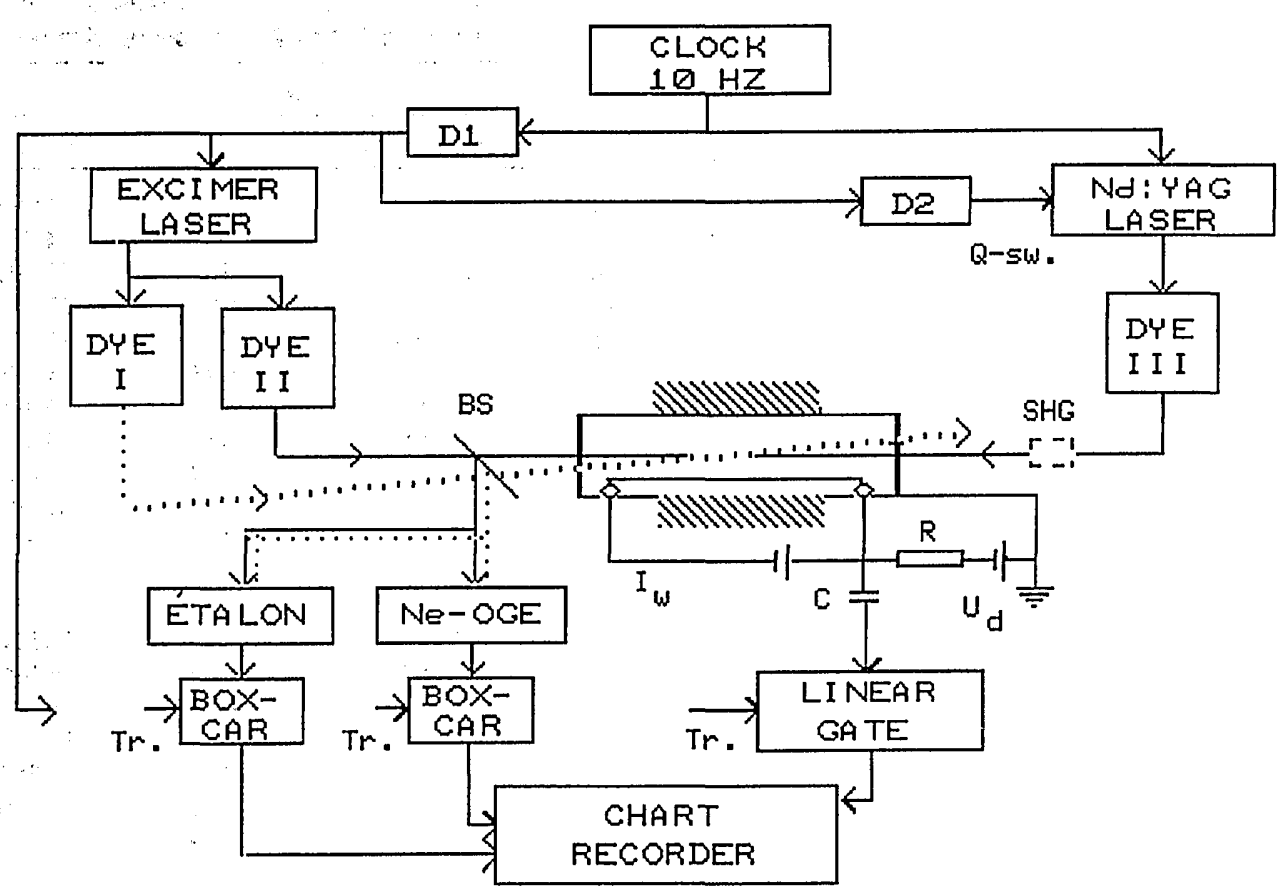

Fig. 1. Block diagram of the experimental arrangement. D1, D2, delays; $Q$-sw., $Q$ switching; BS, beam splitter; Tr's, trigger pulses; SHG, second-harmonic-generation crystal; $U_{d}$, diode voltage; $I_{w}$ wire-heating current; R, resistor; C, coupling capacitor; OGE, Ne optogalvanic spectrum. 


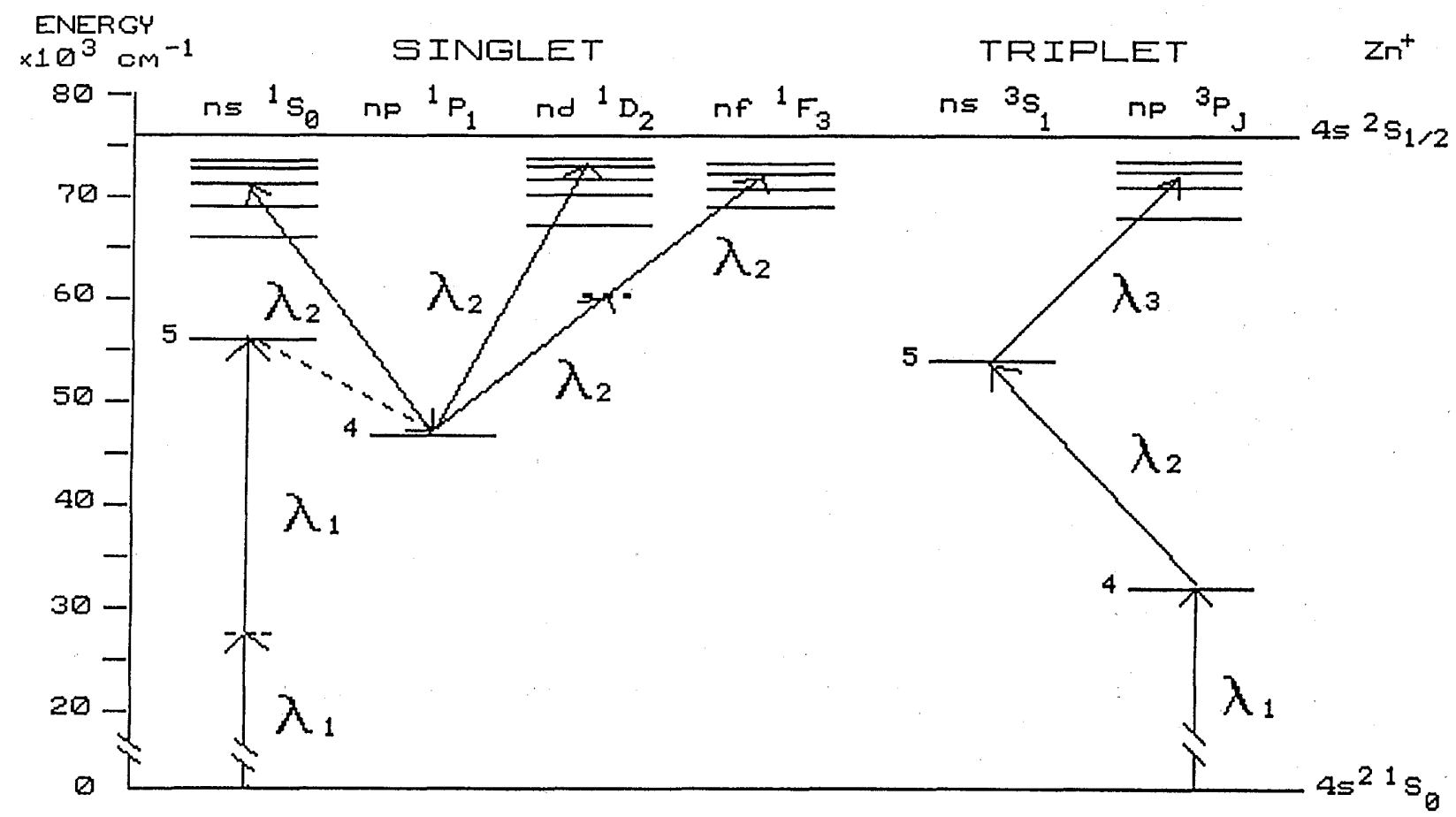

Fig. 2. Part of the $\mathrm{Zn}$ spectrum relevant for this experiment, together with the excitation schemes applied. $\lambda_{i}$ is the wavelength of the $i$ th step laser.

values are presented here for $n=14$ to $n=31$. For $n=5$ to $n=9$, energy levels are known from Moore's tables, ${ }^{8}$ whereas for $n=10$ to $n=12$ they are given by Ref. 6 . For the $n d{ }^{1} D_{2}$ series energy values for $n=12$ to $n=35$ are presented. For $n=4$ to $n=20$ our values differ from those of Ref. 4 by $0.2 \mathrm{~cm}^{-1}$, which is within the accuracy of our experiment.

In the third and the fifth column of Table 1 the quantum defects $\delta$ of the two Rydberg series calculated according to the modified Rydberg formula,

$$
\delta=n-n^{*}=n-[R /(I-E)]^{1 / 2},
$$

are presented. The values for the Rydberg constant $R$ and the threshold $I$ for $\mathrm{Zn}$ are those of Ref. 4 and are $109736.40 \mathrm{~cm}^{-1}$ and $75769.33 \mathrm{~cm}^{-1}$, respectively. The $\delta$ values, plotted versus $n$ in Fig. 3 for the $n s$ and in Fig. 4 for the $n d$ series, show no large variations in $\delta$. Thus the series appear to be unperturbed.

\section{B. Singlet-Triplet $4 s n f$ Spectra}

Only the lowest series members, $4 f^{3} F_{J}^{0}$ (at $68833 \mathrm{~cm}^{-1}$ ), $4 f^{1} F_{3}^{\circ}\left(\right.$ at $\left.68835 \mathrm{~cm}^{-1}\right)$, and $5 f^{1} F_{3}^{\circ}$ (at $71336.15 \mathrm{~cm}^{-1}$ ), are known ${ }^{4}$ thus far. To permit us to record the $n f$ series, the EPD 300 laser induced the first nonresonant transition as above and the TDL50 laser, tunable in the 733-689-nm region, induced the second two-photon $4{ }^{1} P_{1}^{0} \rightarrow n f$ transition (see Fig. 2). The two linearpolarized laser beams were slightly focused in the center of the oven.

The singlet-triplet splitting of the lowest $4 s 4 f$ configuration $^{4}$ is less than $1.5 \mathrm{~cm}^{-1}$. We therefore expect that for the observed $4 s n f$ levels (for $n=8$ to $n=26$ ), which we present here for what is to our knowledge the first time, the two terms merge into each other and cannot be resolved in this investigation. Indeed, the observed spectrum reveals only one peak for each $4 s n f$ configuration, and we attribute to it both singlet and triplet characters because, as is stated in Ref. 4 , the $L S$ coupling starts to break down for the $\mathrm{Zn}$ atom. The series members for $n=6$ and $n=7$ have not been recorded: According to our excitation scheme, wavelengths longer than $733 \mathrm{~nm}$ were needed, which could not be

Table 1. Observed Lines of the Singlet $4 s n s$ and 4 snd Rydberg Series in $\mathrm{Zn}$

\begin{tabular}{llcccc}
\hline & \multicolumn{2}{c}{$4 s n s^{1} S_{0}$} & & \multicolumn{2}{c}{$4 s n d{ }^{1} D_{2}$} \\
\cline { 6 - 6 }$n$ & $E\left(\mathrm{~cm}^{-1}\right)$ & $\delta$ & & $E\left(\mathrm{~cm}^{-1}\right)$ & $\delta$ \\
\hline 12 & & & 74825.0 & 1.220 \\
13 & & & 74978.3 & 1.222 \\
14 & 74920.2 & 2.632 & & 75097.1 & 1.223 \\
15 & 75051.9 & 2.632 & & 75190.8 & 1.228 \\
16 & 75155.1 & 2.634 & & 75266.7 & 1.224 \\
17 & 75237.7 & 2.633 & & 75328.3 & 1.226 \\
18 & 75304.6 & 2.633 & & 75379.3 & 1.226 \\
19 & 75359.7 & 2.633 & & 75422.1 & 1.223 \\
20 & 75405.5 & 2.633 & & 75458.1 & 1.223 \\
21 & 75444.1 & 2.631 & & 75488.7 & 1.225 \\
22 & 75476.7 & 2.635 & 75515.1 & 1.224 \\
23 & 75504.8 & 2.632 & & 75537.9 & 1.225 \\
24 & 75528.9 & 2.636 & 75557.7 & 1.229 \\
25 & 75549.9 & 2.637 & & 75575.1 & 1.231 \\
26 & 75568.4 & 2.630 & & 75590.6 & 1.221 \\
27 & 75584.5 & 2.634 & & 75604.1 & 1.229 \\
28 & 75598.8 & 2.633 & 75616.2 & 1.230 \\
29 & 75611.5 & 2.632 & 75627.1 & 1.223 \\
30 & 75622.8 & 2.634 & 75636.8 & 1.225 \\
31 & 75632.9 & 2.639 & 75645.6 & 1.219 \\
32 & & & 75653.5 & 1.220 \\
33 & & & 75660.6 & 1.231 \\
34 & & & 75667.2 & 1.221 \\
35 & & & 75673.1 & 1.231 \\
\hline & & & &
\end{tabular}




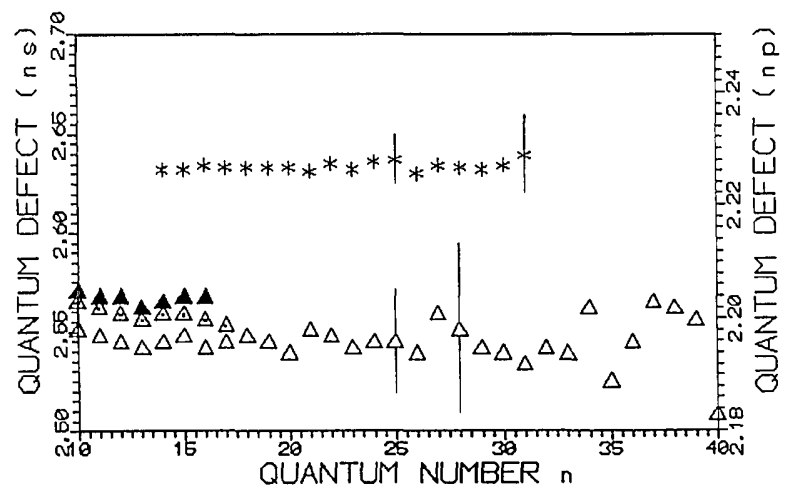

Fig. 3. Quantum-defect plots as a function of the quantum number $n$. Asterisks, 4sns series (left vertical axis); filled triangles, ${ }^{3} P_{0}$ levels; triangles with dots, ${ }^{3} P_{1}$ levels; open triangles, ${ }^{3} P_{2}$ levels of the $4 s n p$ series (right vertical axis). The error bars are according to Eq. (1) for $\Delta E=0.2 \mathrm{~cm}^{-1}$ (experimental accuracy).

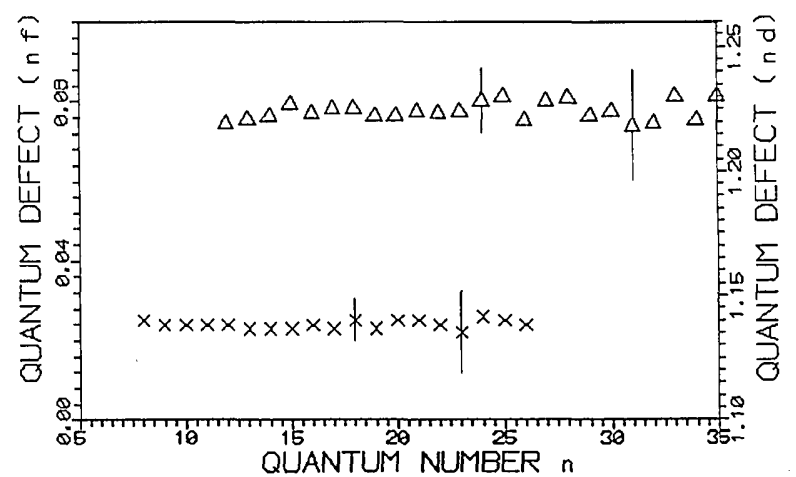

Fig. 4. Same as Fig. 3. Crosses, $4 s n f$ series (left vertical axis); triangles, 4 snd series (right vertical axis).

generated by our tunable laser. The level energies $E_{1}$ of the $4 s n f^{1,3} F_{J}^{0}$ series are listed in Table 2. The calculated quantum defects for this series $(\delta \simeq 0.024)$, in the third column of Table 2, indicate a nonpenetrating orbit and are plotted in Fig. 4. The plot shows that the series is unperturbed.

When we recorded the $n d$ spectra and for $n>16$, weak peaks appeared systematically at the blue side of each strong $n d$ transition (see Fig. 5). The peak energy values were found to agree, within our accuracy, with the $4 s(n-1) f$ level energies, and they are listed, for $n=19$ to $n=26$, in the fourth column of Table 2. Owing to their hydrogenic character, higher $l$ states $(n g, n h$, etc.) are also expected at the same position. Moreover, energy values taken from Ref. 4 show that the $4 s(n+1){ }^{1} P_{1}^{0}$ states lie energetically between the strong $n d$ transitions and their weak satellites but cannot be resolved in Fig. 5 . They may contribute only to the satellites' broadening.

Such weak peaks have also been observed by other authors ${ }^{9-11}$ who used devices similar to ours. Systematic studies ${ }^{9}$ have shown that the ratio of the satellite to the main peak increases both with the vapor and the buffer-gas density but is independent of other experimental conditions. In a semiquantitative model ${ }^{9}$ based on the zero-range Fermi pseudopotential ${ }^{12}$ these authors attributed the satellites to the $l$ mixing of the high Rydberg states through collisions of the electron with neutral perturbers. We have not performed such systematic studies in dependence on the Ar pressure but we note that we have observed the satellites at 6 mbar (4.5 Torr), the highest pressure used in the present experiment. A local dc Stark effect caused by space charges in the diode could be another mechanism for inducing $l$ mixing. Which mechanism is mainly responsible for the observed satellites is still an open question, and it can be resolved in a different experiment.

\section{Triplet $4 s n p$ Spectra}

For the excitation of the $4 s n p^{3} P_{J}^{0}$ states, a three-step excitation scheme has been appointed (see Fig. 2). In the first step the $4 s 4 p{ }^{3} P_{1}^{0}$ state was selectively populated by the TDL50 laser beam, which was set to $6154 \AA$ and frequency doubled in a KDP crystal. The homemade dyelaser beam, counterpropagating to the first beam, was tuned to $4723 \AA$ to induce the second step to the $4 s 5 s{ }^{3} S_{1}$ state. These two laser beams were linearly polarized horizontally. To populate the final $n p$ states, the EPD 300 laser beam crossed the first two beams under a small angle in the center of the oven and was tuned across the 493-452-nm spectral range with the dyes Coumarin 153, 307 , and 102 . The third laser was linearly polarized vertically. The delays between the laser pulses were set appropriately to maximize the signal. The results are listed in Table 3. All three fine-structure components of the $4 s n{ }^{3} P_{J}^{0}$ series have been recorded. Taking into account the polarization directions of the three laser beams, these structures are due to the mixing of the $m_{J}$ sublevels in the intermediate states through collisions. The triplets have been resolved for $n=10$ to $n=17$. For $n=18$ to $n=40$ the three levels merge into one; thus no particular value for $J$ is given in the second column of Table 3 and for the $\delta_{n p}$ value plotted in Fig. 3.

\section{DISCUSSION}

One may estimate the polarizability $\alpha$ of the ionic core from the quantum defect of the nonpenetrating $4 s n f$ series. We consider the two-electron system $4 s n f$ outside closed shells: In the treatment of Van Vleck and

Table 2. Observed Lines of the $4 s n f^{1,3} F_{J}^{0}$ Levels in Zn

\begin{tabular}{|c|c|c|c|c|}
\hline \multirow[b]{2}{*}{$n$} & \multicolumn{2}{|c|}{$2 h v$} & \multicolumn{2}{|c|}{$1 h v$} \\
\hline & $E_{1}\left(\mathrm{~cm}^{-1}\right)$ & $\delta$ & $E_{2}\left(\mathrm{~cm}^{-1}\right)$ & $\left|E_{1}-E_{2}\right|$ \\
\hline 8 & 74044.1 & 0.025 & & \\
\hline 9 & 74407.3 & 0.024 & & \\
\hline 10 & 74666.7 & 0.024 & & \\
\hline 11 & 74858.5 & 0.024 & & \\
\hline 12 & 75004.2 & 0.024 & & \\
\hline 13 & 75117.7 & 0.023 & & \\
\hline 14 & 75207.6 & 0.023 & & \\
\hline 15 & 75280.1 & 0.023 & & \\
\hline 16 & 75339.4 & 0.024 & & \\
\hline 17 & 75388.6 & 0.023 & & \\
\hline 18 & 75429.7 & 0.025 & & \\
\hline 19 & 75464.6 & 0.023 & 75463.7 & 0.9 \\
\hline 20 & 75494.3 & 0.025 & 75494.6 & 0.3 \\
\hline 21 & 75519.9 & 0.025 & 75519.9 & 0.0 \\
\hline 22 & 75542.1 & 0.024 & 75541.9 & 0.2 \\
\hline 23 & 75561.5 & 0.022 & 75561.4 & 0.1 \\
\hline 24 & 75578.4 & 0.026 & 75578.3 & 0.1 \\
\hline 25 & 75593.4 & 0.025 & 75593.5 & 0.1 \\
\hline 26 & 75606.7 & 0.024 & 75606.9 & 0.2 \\
\hline
\end{tabular}




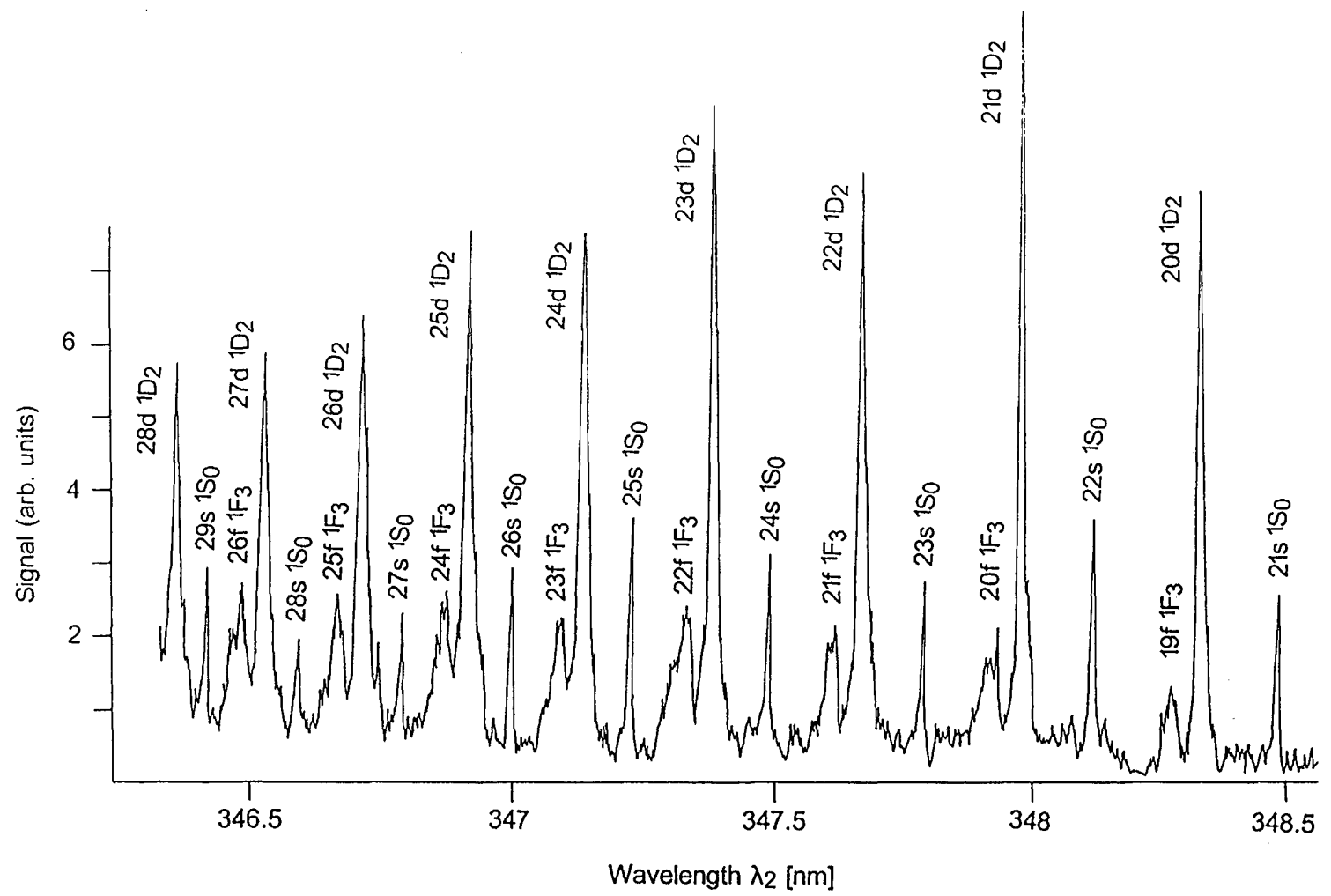

Fig. 5. Part of the 4sns and 4snd Rydberg spectra. The satellites are resolved as weak peaks on the blue wing of the $(n+1) d$ transitions. The experimental conditions are $P_{\mathrm{Ar}}=6 \mathrm{mbar}\left(1 \mathrm{mbar}=7.5 \times 10^{-1}\right.$ Torr $), U_{\text {diode }}=1.5 \mathrm{~V}, T=460^{\circ} \mathrm{C}$.

Whitelaw, ${ }^{13}$ the part of the ionic core that is more easily polarized by the outer, nonpenetrating $n f$ electron is the unexcited $4 s$ (inner) electron. Briefly, in this model one proceeds as follows: The interelectronic term $e^{2} / r_{i j}$ is expanded in multipoles, and first the leading (monopole) term is considered in the Hamiltonian only. This means that one approximates $r_{i j}$ by $r_{o}$, the radius of the outer electron. The Schrödinger equation is then solved for the two independent electrons. In the next approximation the second (dipole) term in the expansion is considered. This implies that terms leading, e.g., to the quadrupole or higher-order polarizabilities are neglected. In this approximation, perturbation theory leads to the following expression for the term energy $W$ :

$$
W=W_{0}-(1 / 2) \alpha e^{2}\left\langle n_{0} l_{o}\left|r^{-4}\right| n_{0} l_{0}\right\rangle,
$$

with

$$
\alpha=-2 \sum_{n} \frac{|\langle 4 s|r| n p\rangle|^{2}}{3 W(4 s \rightarrow n p)}
$$

dipole polarizability. $W_{0}$ has the hydrogenic form $-R Z^{2} / n^{2}$ for nonpenetrating orbits, and $W(4 s \rightarrow n p)$ is the excitation energy of the inner electron to the levels of the principal $n p$ series. The average value $\left\langle r^{-4}\right\rangle$ is the hydrogenic one $\mathrm{e}^{14}$ for $l_{0}=3$.

To estimate $\alpha$ from Eq. (2), we refer to the following simplifications made in the calculations and ask if they are fulfilled for the present case of $\mathrm{Zn}$ : (a) The core must be in an $S$ state, which is obvious for $\mathrm{Zn}$.

(b) The $4 s n f$ singlet-triplet splitting leading to term energy shifts is neglected. In Subsection 3.B we showed that this splitting is negligible and can be ignored.

(c) The total energy $W\left(4 s n f \rightarrow n^{\prime} p n l\right)$ with $l=d$ or $l=g$, which appears in the calculations in the twoindependent-electron approximation, is replaced by the core electron transition energy $W\left(4 s \rightarrow n^{\prime} p\right)$. It turns out that this is a significant approximation, leading to the simple Eq. (2) for $\alpha$.

Since the unperturbed energies of the inner and the outer electrons add to the total energy $W\left(4 s n f \rightarrow n^{\prime} p n l\right)$, approximation (c) implies that the inner-electron transition energies are much larger than the energies of outerelectron transitions to energetically near-lying $n d$ and $n g$ levels. For the present case of the $\mathrm{Zn}^{+}$core the lowest $4 s \rightarrow 4 p^{2} P$ inner-electron transitions is $84880.6 \mathrm{~cm}^{-1}$. Our data show that for $n \cong 20$ the transition energies of the outer electrons to nearby $n d$ states are of the order of some hundred of inverse centimeters, whereas to the still unknown $n g$ states the transition energies are expected to be of the same order of magnitude or less. We therefore conclude that condition (c) is also fulfilled. We apply Eq. (2) for $n=8$ to $n=26$, using the experimental level energies $4 s n f$ in Table 2 , and deduce an average value for $\alpha$,

$$
\alpha=(2.30 \pm 0.12) 10^{-24} \mathrm{~cm}^{3}
$$

or $\alpha=15.54 \alpha_{0}{ }^{3}$ in units of the Bohr radius. 
Table 3. Observed Lines of the Triplet 4snp Rydberg Series in $\mathbf{Z n}$

\begin{tabular}{|c|c|c|c|}
\hline$n$ & $J$ & $E\left(\mathrm{~cm}^{-1}\right)$ & $\delta$ \\
\hline \multirow[t]{3}{*}{10} & 0 & 73963.1 & 2.205 \\
\hline & 1 & 73964.1 & 2.203 \\
\hline & 2 & 73966.6 & 2.198 \\
\hline \multirow[t]{3}{*}{11} & 0 & 74351.0 & 2.204 \\
\hline & 1 & 74351.7 & 2.202 \\
\hline & 2 & 74353.3 & 2.197 \\
\hline \multirow[t]{3}{*}{12} & 0 & 74625.9 & 2.204 \\
\hline & 1 & 74626.5 & 2.201 \\
\hline & 2 & 74627.7 & 2.196 \\
\hline \multirow[t]{3}{*}{13} & 0 & 74828.1 & 2.202 \\
\hline & 1 & 74828.6 & 2.200 \\
\hline & 2 & 74829.4 & 2.195 \\
\hline \multirow[t]{3}{*}{14} & 0 & 74980.8 & 2.203 \\
\hline & 1 & 74981.1 & 2.201 \\
\hline & 2 & 74981.8 & 2.196 \\
\hline \multirow[t]{3}{*}{15} & 0 & 75099.1 & 2.204 \\
\hline & 1 & 75099.4 & 2.201 \\
\hline & 2 & 75099.9 & 2.197 \\
\hline \multirow[t]{3}{*}{16} & 0 & 75192.8 & 2.204 \\
\hline & 1 & 75193.1 & 2.200 \\
\hline & 2 & 75193.5 & 2.195 \\
\hline \multirow[t]{2}{*}{17} & 1 & 75268.4 & 2.199 \\
\hline & 2 & 75268.6 & 2.196 \\
\hline 18 & & 75329.9 & 2.197 \\
\hline 19 . & & 75380.7 & 2.196 \\
\hline 20 & & 75423.2 & 2.194 \\
\hline 21 & & 75458.9 & 2.198 \\
\hline 22 & & 75489.5 & 2.197 \\
\hline 23 & & 75515.8 & 2.195 \\
\hline 24 & & 75538.5 & 2.196 \\
\hline 25 & & 75558.3 & 2.196 \\
\hline 26 & & 75575.7 & 2.194 \\
\hline 27 & & 75590.9 & 2.201 \\
\hline 28 & & 75604.5 & 2.198 \\
\hline 29 & & 75616.6 & 2.195 \\
\hline 30 & & 75627.4 & 2.194 \\
\hline 31 & & 75637.1 & 2.192 \\
\hline 32 & & 75645.8 & 2.195 \\
\hline 33 & & 75653.7 & 2.194 \\
\hline 34 & & 75660.8 & 2.202 \\
\hline 35 & & 75667.4 & 2.189 \\
\hline 36 & & 75673.3 & 2.196 \\
\hline 37 & & 75678.7 & 2.203 \\
\hline 38 & & 75683.7 & 2.202 \\
\hline 39 & & 75688.3 & 2.200 \\
\hline 40 & & 75692.6 & 2.183 \\
\hline
\end{tabular}

To our knowledge this is the first time that the $\mathrm{Zn}^{+}$ dipole polarizability has been obtained experimentally. There are no $a b$ initio theoretical calculations either, but nevertheless we may compare our result with values for $\alpha$ estimated from different experimental data sets and test the validity of the model used above. The polarizability as defined in Eq. (2) may be related to the oscillator strength $f_{i j}$ of the $4 s$ core electron (level $i$ ) to the members $j$ of the $n p$ principal series by the formula ${ }^{15}$

$$
\alpha=\frac{e^{2}}{m} \sum_{j} \frac{f_{i j}}{\omega_{i j}{ }^{2}},
$$

where $\hbar \omega_{i j}=W\left(4 s \rightarrow n^{\prime} p\right)$ and $e$ and $m$, respectively, are the electron charge and mass. Equation (3) indicates that each level $n^{\prime} p$ contributes by an amount $f_{i j} / \omega_{i j}{ }^{2}$ to the total polarizability. With increasing $n^{\prime}$, these contributions decrease rapidly, owing both to the increased transition frequencies $\omega_{i j}$ and to the generally fast-decreasing values of $f_{i j}$. It is then expected that data for the first few $n^{\prime} p$ terms will provide a good estimate of $\alpha$. The contribution of the $4 p^{2} P_{1 / 2,3 / 2}$ levels can be calculated from their lifetime, ${ }^{16} \tau=3 \mathrm{~ns}$, and their energy positions ${ }^{8}$ at 48480.6 and $49354.4 \mathrm{~cm}^{-1}$. This contribution to $\alpha$ is $1.84 \times 10^{-24} \mathrm{~cm}^{3}$; this is already $80 \%$ of our experimental value. So we believe that our value for $\alpha$ confirms the results (for lifetimes) of other authors.

\section{CONCLUSION}

We have measured the energies of the $\mathrm{Zn}$ Rydberg series 4 sns ${ }^{1} S_{0}$ for $n=14$ to $n=31$, 4 snd ${ }^{1} D_{2}$ for $n=12$ to $n=35,4 s n f^{1,3} F_{J}^{o}$ for $n=8$ to $n=26$, and $4 s n p^{3} P_{0,1,2}^{o}$ for $n=10$ to $n=40$. The quantum-defect plots show no noticeable perturbation of these series in the energy range studied. From the $4 s n f$ data we have estimated the $\mathrm{Zn}^{+}(4 s)$ dipole polarizability $\alpha$, which is in good agreement with lifetime measurements from other authors.

\section{REFERENCES}

1. P. Esherick, J. A. Armstrong, R. W. Dreyfus, and J. J. Wynne, Phys. Rev. Lett. 36, 1296-1299 (1976).

2. J. A. Armstrong, P. Esherick, and J. J. Wynne, Phys. Rev. A 15, 180-196 (1977)

3. W. C. Martin and V. Kaufman, J. Opt. Soc. Am. 60, 1096-1099 (1970).

4. C. M. Brown, S. G. Tilford, and M. L. Ginter, J. Opt. Soc. Am. 65, 1404-1409 (1975).

5. A. L. Osherovich, Y. F. Verolainen, and V. I. Privalov, Opt. Spectrosc. (USSR) 46, 617-619 (1979).

6. M. Chantepie, J. L. Cojan, J. Landais, B. Laniepce, A. Moudden, and M. Aymar, Opt. Commun. 51, 391-395 (1984).

7. A. R. Striganov and N. S. Sventitskii, Tables of Spectral Lines of Neutral and Ionized Atoms (Plenum, New York, 1968).

8. C. E. Moore, Atomic Energy Levels, Natl. Standards Ref. Data Ser. Natl. Bur. Standards 35/V.II, 124-126 (1971).

9. J.-Y. Zhang and K. T. Lu, J. Phys. B 20, 5065-5077 (1987).

10. D.-H. Wu, Y.-F. Yang, and K. T. Lu, J. Phys. B 23, L149-L152 (1990).

11. Y. Makdisi and K. S. Bhatia, Can. J. Phys. 68, 1464-1468 (1990).

12. A. Omont, J. Phys. (Paris) 38, 1343-1359 (1977).

13. J. H. Van Vleck and N. G. Whitelaw, Phys. Rev. A 44, $551-569$ (1933).

14. H. A. Bethe and E. E. Salpeter, Quantum Mechanics of Oneand Two-Electron Atoms (Springer-Verlag, Berlin, 1957), Chap. 1, p. 17.

15. I. I. Sobelman, Atomic Spectra and Radiative Transitions (Springer-Verlag, Berlin, 1979), Chap. 9, p. 209.

16. T. Andersen, O. Poulsen, and P. S. Ramanujam, J. Quantum Spectros. Radiat. Transfer 16, 521-527 (1976). 